\title{
Dimension estimation for autonomous nonlinear systems
}

\author{
Alberto Padoan and Alessandro Astolfi
}

\begin{abstract}
The problem of estimating the dimension of the state-space of an autonomous nonlinear systems is considered. Assuming that sampled measurements of the output and finitely many of its time derivatives are available, an iterative algorithm able to retrieve the dimension of the minimal state-space realization is derived. The performance of the proposed algorithm are evaluated on benchmark nonlinear systems.
\end{abstract}

\section{INTRODUCTION}

Determining the dimension of the state space from experimental observations is a crucial step in the mathematical modelling of dynamical systems. Among the infinitely many state space descriptions of a dynamical system, it is desirable to find the dimension of the smallest one. Conceptually, the dimension of the state space can be thought as a measure of the complexity of a dynamical system. The difficulty of the problem of estimating the dimension of the state space of a dynamical system is related to the properties of the class of dynamical systems considered. For example, the dimension of the state space space of a linear system can be recovered by means of subspace identification methods [1-4], provided the measured input-output data are not too noisy. Understanding the properties of nonlinear dynamical systems from experimental observations is more difficult, even under simplifying assumptions [5].

A popular approach to state space reconstruction for autonomous nonlinear systems is based on the "method of delays" [6]. This method hinges upon the fact that finitely many functions of the output of an autonomous nonlinear system can be used to build vectors which lie generically on an embedded manifold of the original state space, provided that the selected functions are more than twice the dimension of the state space of the system. Due to their practical accessibility, time-delayed versions of the output are often chosen as such functions (hence the name method of delays). This method of state space reconstruction has led several authors to disregard the problem of determining the dimension of the state space of the system, but to estimate the minimum embedding dimension

A. Padoan is with the Department of Electrical and Electronic Engineering, Imperial College London, London SW7 2AZ, UK (e-mail: alberto.padoan130imperial.ac.uk). A. Astolfi is with the Department of Electrical and Electronic Engineering, Imperial College London, London SW7 2AZ, UK and with the Dipartimento di Ingegneria Civile e Ingegneria Informatica, Università di Roma "Tor Vergata", Via del Politecnico 1, Rome 00133, Italy (e-mail: a.astolfi@imperial.ac.uk). instead, i.e. the minimum number of time-delayed versions of the output needed to describe a state space in a higher-dimensional manifold. The computation of the minimum embedding dimension is usually carried out with the method of false nearest neighbours [7]. The reader is referred to [5], and references therein, for further detail.

In some special situations the dimension of the (portion of the) state space can be reconstructed by visual inspection (see, for an example, [8]). The dimension of the state space of the system, however, may not coincide with the dimension of the geometric objects obtained with this method. In addition, the determination of the dimension by visual inspection is not possible for systems of dimension greater than three. In general, the lack of quantitative arguments makes visual inspection extremely subjective and thus not suitable to provide reliable estimates of the dimension of the state space.

The main contribution of this work is an iterative algorithm which estimates the dimension of the state space of an autonomous systems from measurements of the output and finitely many of its time derivatives. The proposed approach hinges upon a local observability assumption and is partly inspired by the subspace approach to linear system identification [1-4]. Under the assumption that the sampling operation is sufficiently fast, an approximate linear relationship between the measured data can be derived. This relationship is used as a test condition and to derive an iterative algorithm to estimate the dimension of the state space of the system. Simulation results show that, if the sampling period is sufficiently small, the dimension of the state space of the system can be correctly estimated.

The remainder of the paper is organized as follows. Section II defines the problem and introduces basic assumptions. Section III illustrates the main results, including an iterative algorithm which estimates the dimension of the state space from measured output data. In Section IV simulation results show the effectiveness of the proposed algorithm on benchmark nonlinear systems. Practical considerations and future directions of research are discussed in Section V.

Notation: Standard notation is used. $\mathbb{R}, \mathbb{R}^{n}$ and $\mathbb{R}^{p \times m}$ denote the set of real numbers, of $n$-dimensional vectors with real entries, and of $p \times m$-dimensional matrices with real entries, respectively. The Schur comple- 
ment in the partitioned real matrix

$$
A=\left[\begin{array}{ll}
A_{11} & A_{12} \\
A_{21} & A_{22}
\end{array}\right] \in \mathbb{R}^{i \times j},
$$

with $1<i \leq j$, of the leading square submatrix $A_{11} \in \mathbb{R}^{k \times k}$, with $k \in[1, i-1]$, is defined as

$$
A / A_{11}=A_{22}-A_{21} A_{11}^{-1} A_{12} \in \mathbb{R}^{(i-k) \times(j-k)},
$$

provided that the matrix $A_{11}$ is non-singular. The symbol $\|\cdot\|_{2}$ is used to denote the standard Euclidean norm, defined as $\|x\|_{2}=\left(\sum_{i=1}^{n} x_{i}^{2}\right)^{1 / 2}$ for all $x=$ $\left(x_{1}, x_{2}, \ldots, x_{n}\right) \in \mathbb{R}^{n}$, and the corresponding induced operator norm. The notation $y^{(k)}(t)$, with $k$ a positive integer, is used to denote the $k$-th order derivative of the function $y$ at time $t$, provided it exists. The Lie derivative of the smooth function $h$ along the smooth vector field $f$ is defined as $L_{f} h=\frac{\partial h}{\partial x} f$, and the functions $L_{f}^{k} h$, with $k$ a non-negative integer, are defined recursively as $L_{f}^{k+1} h=L_{f}\left(L_{f}^{k} h\right)$, with $L_{f}^{0} h=h$.

\section{Problem formulation}

Consider a continuous-time, autonomous, nonlinear system described by equations of the form ${ }^{1}$

$$
\dot{x}=f(x), \quad y=h(x),
$$

in which $x(t) \in \mathbb{R}^{n}$ and $y(t) \in \mathbb{R}$ denote the unknown state and the measured output of the system, respectively. Assume, without loss of generality, that the state of the system evolves on an open set $\mathcal{X} \subseteq \mathbb{R}^{n}$ containing the (unknown) initial condition $x_{0} \in \mathbb{R}^{n}$ for all $^{2} t \geq 0$.

The dimension estimation problem can be formulated as follows. Suppose that system (1) is unknown, and that the output signal $y$ is known only through its $j$ time samples at the time instants $t_{1}, t_{2}, \ldots, t_{j} \in \mathbb{R}$, with $0<t_{1}<t_{2}<\ldots<t_{j}$. Assume that reliable estimates of the first $i-1$ time derivatives $y^{(1)}\left(t_{k}\right), y^{(2)}\left(t_{k}\right), \ldots, y^{(i-1)}\left(t_{k}\right)$ of the output $y(t)$ at time $t_{k}$ are also available for each integer $k \in[1, j]$. The goal is to determine the dimension of the unknown system (1), i.e. the positive integer $n$.

Remark 1. Although differentiation is an ill-posed operation, the assumption that the output $y$ and finitely many of its time derivatives are available is only fairly restrictive. Efficient algorithms which implement numerical differentiation schemes are widely employed by the control community $[9,10]$. An in-depth discussion of the issue of computing the derivatives of continuous-time variables which are known only through their time samples is given in [11].

\footnotetext{
${ }^{1}$ The mappings $f$ and $h$ are assumed to be smooth, i.e. $C^{\infty}$, and such that $f(0)=0$ and $h(0)=0$.

${ }^{2}$ Similar considerations can be performed when the state of the system is only defined on a real interval of the form $\left[0, t_{\max }\right)$, with $t_{\max }>0$.
}

To streamline the presentation of our results we define the vector

$$
Y_{k}(t)=\left[\begin{array}{c}
y(t) \\
y^{(1)}(t) \\
\vdots \\
y^{(k-1)}(t)
\end{array}\right] \in \mathbb{R}^{k}
$$

with $k$ a positive integer, for all $t \geq 0$. We also introduce the matrix

$$
Y=\left[\begin{array}{llll}
Y_{i}\left(t_{1}\right) & Y_{i}\left(t_{2}\right) & \ldots & Y_{i}\left(t_{j}\right)
\end{array}\right] \in \mathbb{R}^{i \times j} .
$$

The matrix $Y$ is referred to as the output matrix. Note that only available data are required to construct it. With the notation introduced above, the dimension estimation problem can be reformulated as finding the positive integer $n$ based on the knowledge of the output matrix $Y$.

Before proceeding further, we now introduce some auxiliary assumptions.

Assumption 1. The positive integers $i$ and $j$ are such that $n<i \leq j$.

Note that this assumption requires an upper bound on $n$ to be known. In practice, since there is no finite procedure to find an upper bound of $n$, it is necessary to assume an upper bound a priori.

Assumption 2. The sampling time instants $t_{1}, t_{2}, \ldots, t_{j}$ are equidistant, i.e. $t_{k}=k T$ for each $k \in[1, j]$, with $T>0$.

This assumption is not necessary, but simplifies the presentation. $T$ is referred to as the sampling period and represents the distance between two consecutive sampling instants. Without loss of generality, $T$ is assumed to be a small positive number that can be assigned.

Assumption 3. Consider the system (1). The mapping $H: \mathcal{X} \rightarrow \mathbb{R}^{n}$, defined as

$$
H(x)=\left[\begin{array}{c}
h(x) \\
L_{f} h(x) \\
\vdots \\
L_{f}^{n-1} h(x)
\end{array}\right],
$$

for all $x \in \mathcal{X}$, is a local diffeomorphism ${ }^{3}$ (onto its image) on a suitable open subset $\mathcal{X}_{0}$ of $\mathcal{X}$ containing the initial condition $x_{0}$.

Assumption 3 is not particularly restrictive, since it is satisfied by a vast number of nonlinear systems (see [12] for some examples). In addition, without an observability assumption, the unobservable part of the state trajectory cannot be recovered exactly. In linear system identification, where not only the order but also the system matrices describing a multiple-input

\footnotetext{
${ }^{3} \mathrm{~A}$ smooth map $H$ is a diffeomorphism if it is one-to-one and onto, with a smooth inverse map $H^{-1}$.
} 
multiple-output system have to be estimated, the observability of the system to be identified is always assumed [1-4]. As a result, Assumption 3 can be regarded as a nonlinear counterpart of the standard observability assumption, and it coincides with the linear notion of observability when the system is linear.

Assumption 3 has important consequences on the structure of system (1). The mapping (2) qualifies as a change of coordinates, locally defined around the initial condition $x_{0}$, such that, in the new coordinates, the dynamics of system (1) are described by equations of the form

$$
\begin{aligned}
\dot{\chi}_{1} & =\chi_{2}, \\
& \vdots \\
\dot{\chi}_{n-1} & =\chi_{n}, \\
\dot{\chi}_{n} & =\phi_{n}\left(\chi_{1}, \ldots, \chi_{n}\right), \\
y & =\chi_{1} .
\end{aligned}
$$

Recalling that the origin is an equilibrium point, the mapping $\phi_{n}: \mathbb{R}^{n} \rightarrow \mathbb{R}^{n}$ can be written, without loss of generality, as

$$
\phi_{n}(\chi)=\alpha_{1}(\chi) \chi_{1}+\ldots+\alpha_{n}(\chi) \chi_{n}
$$

in which the functions $\alpha_{k}: \mathbb{R}^{n} \rightarrow \mathbb{R}$ are (possibly nonunique) smooth functions. Thus, since $i>n$, the use of the local coordinates in (3) allows to obtain the relation

$$
Y_{i}(t)=\left[\frac{I}{M_{i}\left(Y_{n}(t)\right)}\right] Y_{n}(t)
$$

for all $t \geq 0$, in which $M_{i}: \mathbb{R}^{n} \rightarrow \mathbb{R}^{(i-n) \times n}$ is a smooth matrix-valued mapping. In other words, the vector $Y_{i}$ can be obtained through a time-varying linear transformation of the vector $Y_{n}$, determined by the matrix $M_{i}$. The relationship in (3) is instrumental to derive the iterative algorithm proposed in the next section.

\section{MAIN RESUltS}

In this section an iterative algorithm which solves the dimension estimation problem is presented. For sufficiently small values of the sampling period $T$, the algorithm extracts an estimate of the dimension of the system from the output matrix $Y$. Before illustrating the algorithm, it is instructive to re-consider the dimension estimation problem for linear systems.

\section{A. Linear systems ${ }^{4}$}

Consider a continuous-time, autonomous, linear system described by equations of the form

$$
\dot{x}=A x, \quad y=C x,
$$

in which $x(t) \in \mathbb{R}^{n}$ and $y(t) \in \mathbb{R}$. Autonomous linear systems can be regarded as a special class of systems of the form (1) in which the mappings $f$ and $h$ are linear 9].

\footnotetext{
${ }^{4}$ This subsection has been partly inspired and motivated by [4, Ch.
}

functions of the state. In view of Assumption 3, the observability matrix

$$
\Gamma_{i}=\left[\begin{array}{c}
C \\
C A \\
\vdots \\
C A^{i-1}
\end{array}\right] \in \mathbb{R}^{i \times n},
$$

is full rank. In addition, by the linearity of the system, the output matrix $Y$ can be written as

$$
Y=\Gamma_{i} X_{j}
$$

where the matrix $X_{j}$ is defined as

$$
X_{j}=\left[\begin{array}{llll}
x\left(t_{1}\right) & x\left(t_{2}\right) & \cdots & x\left(t_{j}\right)
\end{array}\right] \in \mathbb{R}^{n \times j} .
$$

If $X_{j}$ is full $\mathrm{rank}^{5}$, then the condition

$$
\operatorname{rank}(Y)=n
$$

holds by Sylvester's inequality ${ }^{6}$. Equation (4) shows that a necessary condition for a system to be linear, or more precisely to have a linear realization, is the finiteness of the rank of the output matrix $Y$. In this special case, under mild assumptions, the dimension of the system coincides with the rank of the output matrix $Y$. The finiteness of the rank of the output matrix $Y$ is necessary, though not sufficient for a system to be linear. In fact, there exist "truly" nonlinear systems of the form (1) for which the output matrix $Y$ has finite rank, as shown in the next example.

Example 1. [14] Consider the nonlinear system described by equations of the form

$$
\dot{x}=f(x), \quad y=h(x),
$$

in which the state is $x(t)=\left(x_{1}(t), x_{2}(t), x_{3}(t)\right) \in \mathbb{R}^{3}$, the output is $y(t) \in \mathbb{R}$, the system mappings are defined as

$$
f(x)=\left[\begin{array}{lll}
x_{2}^{3} & x_{3} & 0
\end{array}\right]^{\top}, \quad h(x)=x_{1},
$$

for all $x \in \mathbb{R}^{3}$, and the initial condition of the state is $x(0)=x_{0} \in \mathbb{R}^{3} \backslash\{0\}$. For this system the mapping $H(x)$ defined as in (2) is a diffeomorphism in any open subset of $\mathbb{R}^{3}$ which does not contain the origin. Thus, the following considerations hold as long as the state of the system evolves away from the origin.

A first peculiarity of system (5) is that the rank of any associated output matrix $Y$ is always finite. To see this, observe that the $k$-th Lie derivative $L_{f}^{k} h(x)$ of the output function $h(x)$ is identically zero for all $k \geq 5$. This, in turn, implies that the $k$-th time derivative of

\footnotetext{
${ }^{5}$ Controllability is a generic property [13]: if a pair of matrices $(A, B) \in \mathbb{R}^{n \times n} \times \mathbb{R}^{n \times m}$ is considered as a point in a finitedimensional space, the set of controllable pairs is open and dense in the whole space. Thus, it is not restrictive to assume the controllabilty of the pair $(A, x(t))$, which, in turn, implies that the state matrix $X_{j}$ is full rank. Note also that if the pair $(A, x(0))$ is controllable, then the pair $(A, x(t))$ is controllable for all $t \geq 0$ finite.

${ }^{6}$ See Lemma 2.1 in $[4$, p. 16$]$.
} 
the output signal $y^{(k)}(t)$ is also identically zero for all $k \geq 5$. By definition, only the first 5 rows of the vectors $Y_{k}(t)$ can be non-zero for each $k \geq 5$. As a result the output matrix $Y$ associated with system (5) has at most rank 5, regardless of the choice of the sampling instants.

Another interesting aspect of system (5) is that its dynamics can be described by a higher-dimensional observable linear system. To see this consider the mapping $\psi: \mathbb{R}^{3} \rightarrow \mathbb{R}^{5}$, defined as

$$
\psi(x)=\left[\begin{array}{lllll}
x_{1} & x_{2}^{3} & 3 x_{2}^{2} x_{3} & 6 x_{2} x_{3} & 6 x_{3}
\end{array}\right]^{\top},
$$

for each $x \in \mathbb{R}^{3}$. By defining the auxiliary variable $\xi=\psi(x)$, it is easy to see that the dynamics of the nonlinear system (5) can be described by a (nilpotent) linear realization of the form

$$
\dot{\xi}=F \xi, \quad y=H \xi,
$$

if the initial condition is set to $\xi(0)=\psi\left(x_{0}\right) \in \mathbb{R}^{5} \backslash\{0\}$, and the constant matrices $F \in \mathbb{R}^{5 \times 5}$ and $H \in \mathbb{R}^{1 \times 5}$ are chosen, for example, as

$$
\begin{aligned}
F & =\left[\begin{array}{lllll}
0 & 1 & 0 & 0 & 0 \\
0 & 0 & 1 & 0 & 0 \\
0 & 0 & 0 & 1 & 0 \\
0 & 0 & 0 & 0 & 1 \\
0 & 0 & 0 & 0 & 0
\end{array}\right], \\
H & =\left[\begin{array}{lllll}
1 & 0 & 0 & 0 & 0
\end{array}\right] .
\end{aligned}
$$

This shows that, in a 5-dimensional space, the nonlinear system (5) can be seen as an observable nilpotent linear system. Note, however, that the linear realization (6) yields more output trajectories than the nonlinear realization (5). In other words, only certain initial conditions of the linear system (6) yield the state trajectories produced by the nonlinear system (5).

The existence of a higher-dimensional linear realization of the nonlinear system (5) is related to the fact that its observation space has finite dimension. More precisely, a necessary and sufficient condition for an autonomous nonlinear system to be immersed into a linear observable system is to have a finite-dimensional observation space. The reader is referred to [12, Ch. 8] for further detail.

Remark 2. In discrete-time subspace identification the linearity of the system to be identified is typically assumed a priori. The finiteness of the rank of the discrete-time counterpart of the output matrix $Y$ may be seen in that context as a confirmation of the linearity of the underlying system. The example above, however, reveals that this conclusion is at times misleading: of fact there exist nonlinear systems with an associated finite rank output matrix $Y$ (and an observable linear realization).

Remark 3. Consider a system described by equations of the form (1) of dimension $n \geq 1$. Note that the $k$-th time derivative $y^{(k)}(t)$ of the output $y(t)$ can be expressed as a linear combination (over $\mathbb{R}$ ) of the signals $y(t), y^{(1)}(t), \ldots, y^{(k-1)}(t)$ only if the system admits a linear realization of dimension $k$. This implies that all the principal submatrices of the output matrix $Y$ of order $k \in[1, n]$ are non-singular if the system is "truly" nonlinear.

\section{B. Nonlinear systems}

Consider the nonlinear system (1). In view of equation (3), the expression

$$
Y_{i}\left(t_{k}\right)=\left[\frac{I}{M_{i}\left(Y_{n}\left(t_{k}\right)\right)}\right] Y_{i}\left(t_{k}\right),
$$

holds for each $k \in[1, j]$. By continuity of $M_{i}$ with respect to its argument, the output matrix $Y$ can be written as

$$
Y=\left[\begin{array}{c|c}
I & 0 \\
\hline M_{0} & 0
\end{array}\right] Y+O(T)
$$

since

$$
Y_{k}=\left[\begin{array}{c|c}
I & 0 \\
\hline M_{0} & 0
\end{array}\right] Y_{k}+\left[\begin{array}{c|c}
0 & 0 \\
\hline M_{k}-M_{0} & 0
\end{array}\right] Y_{k}
$$

and

$$
\lim _{T \rightarrow 0} M_{k}=M_{0} .
$$

Consider now the family of partitions of the output matrix $Y$, parameterized by the positive integer $k$, defined as

$$
Y=\left[\begin{array}{c|c}
Y_{11}(k) & Y_{12}(k) \\
\hline Y_{21}(k) & Y_{22}(k)
\end{array}\right],
$$

with $Y_{11}(k) \in \mathbb{R}^{k \times k}, Y_{12}(k) \in \mathbb{R}^{k \times(j-k)}, Y_{21}(k) \in$ $\mathbb{R}^{(i-k) \times k}$, and $Y_{22}(k) \in \mathbb{R}^{(i-k) \times(j-k)}$ for each integer $k \in[1, i-1]$. In each partition, the parameter $k$ represents the order of the principal submatrix $Y_{11}(k)$. The number of rows and columns of the other matrices is determined correspondingly. For example, if $k=1$, the output matrix $Y$ is partitioned as

$\left[\begin{array}{c|ccc}y\left(t_{1}\right) & y\left(t_{2}\right) & \ldots & y\left(t_{j}\right) \\ \hline y^{(1)}\left(t_{1}\right) & y^{(1)}\left(t_{2}\right) & \ldots & y^{(1)}\left(t_{j}\right) \\ \vdots & \vdots & \vdots & \vdots \\ y^{(i-1)}\left(t_{1}\right) & y^{(i-1)}\left(t_{2}\right) & \ldots & y^{(i-1)}\left(t_{j}\right)\end{array}\right]$,

and

$$
\begin{aligned}
& Y_{11}(1)=y\left(t_{1}\right), \\
& Y_{12}(1)=\left[\begin{array}{lll}
y\left(t_{2}\right) & \ldots & y\left(t_{j}\right)
\end{array}\right], \\
& Y_{21}(1)=\left[\begin{array}{c}
y^{(1)}\left(t_{1}\right) \\
\vdots \\
y^{(i-1)}\left(t_{1}\right)
\end{array}\right] \\
& Y_{22}(1)=\left[\begin{array}{ccc}
y^{(1)}\left(t_{2}\right) & \cdots & y^{(1)}\left(t_{j}\right) \\
\vdots & \vdots & \vdots \\
y^{(i-1)}\left(t_{2}\right) & \cdots & y^{(i-1)}\left(t_{j}\right)
\end{array}\right] \text {. }
\end{aligned}
$$


Using this family of partitions, condition (7) can be rewritten as

$$
\begin{aligned}
& Y_{21}(n)=M_{0} Y_{11}(n)+O(T), \\
& Y_{22}(n)=M_{0} Y_{12}(n)+O(T) .
\end{aligned}
$$

If $Y_{11}(n)$ is non-singular, the above equations imply

$$
\left\|Y / Y_{11}(n)\right\|_{2}=O(T) .
$$

In other words, if the sampling period $T$ is sufficiently small and $Y_{11}(n)$ is non-singular, the Schur complement of the principal submatrix $Y_{11}(n)$ in the output matrix $Y$ is infinitesimal of order $O(T)$. Observe that if $k<n$ the approximation (9) does not hold for the Schur complement $Y / Y_{11}(k)$. The minimum value of the parameter $k$ in $Y / Y_{11}(k)$ for which the approximation (9) holds is exactly the dimension of the system. Note, however, that $n$ may not necessarily be the unique value of $k$, because there may be "larger" state-space descriptions of the same system, as seen in Example 1.

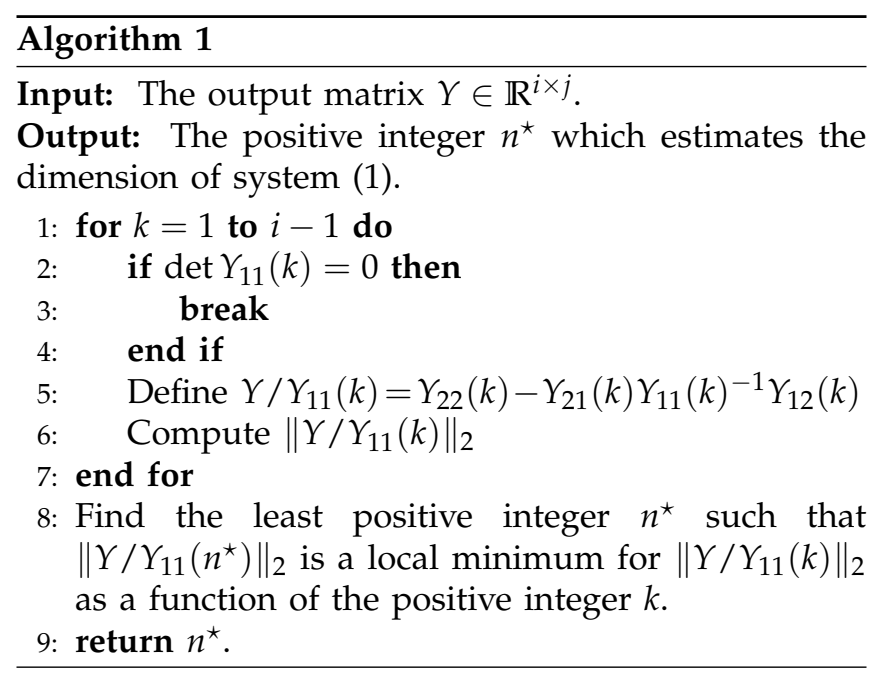

The approximation stated in (9) allows to devise an iterative algorithm which estimates the dimension of the system. The algorithm is described below and given in pseudo-code in Algorithm 1. The idea is to find the least positive integer in the partition (8) such that (9) holds. Using an exhaustive search approach, the dimension of the system is found by increasing $k$, and repeatedly testing if condition (9) holds. For convenience, we introduce the auxiliary matrix

$$
Y / Y_{11}(k)=Y_{22}(k)-Y_{21}(k) Y_{11}(k)^{-1} Y_{12}(k),
$$

with $k \in[1, i-1]$, provided that $Y_{11}(k)$ is nonsingular. At each iteration the distance between the matrix $Y / Y_{11}(k)$ and the zero matrix is measured by the 2-norm $\left\|Y / Y_{11}(k)\right\|_{2}$. From a computational point of view, this amounts to calculating the largest singular value of the matrix $Y / Y_{11}(k)$. The least positive integer $k$ such that $\left\|Y / Y_{11}(k)\right\|_{2}$ reaches a local minimum is then found by inspection. For sufficiently small $T$, such value of $k$ coincides with the dimension of the system, i.e. $k=n$.

The invertibility of the $k$-th leading submatrix $Y_{11}(k)$, with $k \in[1, i-1]$, of the output matrix $Y$ is necessary to define the auxiliary matrix $Y / Y_{11}(k)$. This condition must be tested before doing any computation at each iteration. In practice, there may be values of $k$ for which the matrix $Y_{11}(k)$ is singular. In particular, when the system admits a linear realization and $k \geq n$, the corresponding $Y_{11}(k)$ is singular, as seen in Example 1. Another reason for $Y_{11}(k)$ to be singular is the selection of a too small sampling period, as detailed in the next section. In any of these situations, if at iteration $k$ the matrix $Y_{11}(k)$ is singular, the algorithm is stopped.

Finally, note that the effectiveness of Algorithm 1 can be severely undermined by the choice of its parameters. If $T$ is not sufficiently small, or the amount of available data is not sufficient, i.e. the condition $n<i \leq j$ is not satisfied, the algorithm may determine a wrong estimate of the dimension of the system.

\section{Simple Numerical EXAmples}

We now present some numerical results showing the performance of Algorithm 1 on system (5) and other benchmark nonlinear systems. The equations of the dynamical systems considered and the numerical parameters used in simulations are reported in Table I. Simulations are performed using an explicit RungeKutta $(2,3)$-order integration method, and a normally distributed pseudo-random initial condition. The integration step used in the simulations of system (5) and the other systems are $10^{-4} \mathrm{~ms}$ and $10^{-6} \mathrm{~ms}$, respectively. To make the simulations more realistic, cascades of continuous-time filters with transfer function of the form

$$
W(s)=\frac{s}{1+s T_{d}},
$$

with $T_{d}>0$, the so-called "rough differentiators", are used to filter the output signal $y$ to obtain estimates of its high-order time derivatives. In other simulations, the needed time derivatives of the output signal $y$ have been computed analytically using a symbolic software package Maple. The results obtained are similar to the ones reported herein, and thus omitted.

In the first simulation, $i=15$ and $j=5000$. The output samples are taken in the time interval $t \in[0,0.5] \mathrm{s}$. The sampling time for the measurements of the output signal is $T=0.1 \mathrm{~ms}$. The parameter $T_{d}$ of the rough differentiators is set to $T_{d}=20$ and $T_{d}=100$, when simulating system (5) and the other systems, respectively. Figure 2 displays the 2-norm $\left\|Y / Y_{11}(k)\right\|_{2}$ of the matrix $Y / Y_{11}(k)$ as a function of the iteration $k$ of Algorithm 1 for the system (5), for the Duffing oscillator, the pendulum, and the van der Pol oscillator, respectively. Note that the plots are in logarithmic scale and normalized to the maximum value. Figure 2 shows that Algorithm 1 correctly estimates the dimension of 
TABLE I: Test dynamical systems

\begin{tabular}{|c|c|c|c|}
\hline System & \multicolumn{2}{|r|}{ Equations } & Parameters \\
\hline System (5) & & $\begin{aligned} \frac{d x_{1}}{d t} & =x_{2}^{3} \\
\frac{d x_{2}}{d t} & =x_{3} \\
\frac{d x_{3}}{d t} & =0 \\
y & =x_{1}\end{aligned}$ & none \\
\hline Duffing oscillator & $\begin{array}{l}\frac{d x_{1}}{d t} \\
\frac{d x_{2}}{d t} \\
y\end{array}$ & $\begin{array}{l}=x_{2} \\
=-\alpha x_{1}-\beta x_{1}^{3}-\delta x_{2} \\
=x_{1}\end{array}$ & $\begin{array}{l}\alpha=1 \\
\beta=1 \\
\delta=0\end{array}$ \\
\hline Pendulum & $\begin{array}{l}\frac{d x_{1}}{d t} \\
\frac{d x_{2}}{d t} \\
y\end{array}$ & $\begin{array}{l}=x_{2} \\
=-\frac{g}{l} \sin x_{1}-\frac{k}{m} x_{2} \\
=x_{1}\end{array}$ & $\begin{array}{l}g=9.8 \\
l=9.8 \\
k=0 \\
m=1\end{array}$ \\
\hline van der Pol oscillator & $\begin{array}{l}\frac{d x_{1}}{d t} \\
\frac{d x_{2}}{d t} \\
y\end{array}$ & $\begin{array}{l}=x_{2} \\
=-x_{1}+\mu\left(1-x_{1}^{2}\right) x_{2} \\
=x_{1}\end{array}$ & $\mu=0.2$ \\
\hline
\end{tabular}

the considered systems. The least positive integer $k$ for which $\left\|Y / Y_{11}(k)\right\|_{2}$ has a local minimum as a function of the iterations of Algorithm 1 is exactly the dimension of these systems.

In the second simulation, the experimental setup is unchanged. The measured data are separated in five smaller batches corresponding to five consecutive moving windows. Each batch is processed separately and used to create an output matrix to be given as input to Algorithm 1. The rounded integer value of the average value predicted by Algorithm 1 for each batch is then taken as the sought estimate of the dimension. In principle, this operation enforces robustness against the errors introduced by the approximations made. Figure 1 displays the 2-norm $\left\|Y / Y_{11}(k)\right\|_{2}$ of the matrix $Y / Y_{11}(k)$ as a function of the iterations of Algorithm 1 for the system (5) (top-left), the Duffing oscillator (topright), the pendulum (bottom-left), and the van der Pol oscillator (bottom-right). It can be noted that for system (5), the runs of Algorithm 1 give incongruous results. In the first simulation, where all the available information is used in a single run, a clearer indication of the dimension of the system is obtained. This is probably due to the intrinsic ambiguity of system (5), which admits both a linear and a nonlinear realization. In all other cases, the dimension of the system is correctly estimated by Algorithm 1.

\section{Conclusion}

The problem of estimating the dimension of the state space of nonlinear autonomous systems has been studied. An iterative algorithm which determines the dimension of the state space of the system from given sampled measurements of the output and a finite number of its time derivatives has been proposed. The algorithm has been evaluated on benchmark nonlinear systems and a more complex case study.

A number of questions and research directions are left open. Implementative aspects, such as effectively sampling the needed quantities, should be investigated. A multivariable version of the presented results should be developed. Finally, the effect of noise should be explored, to account for modelling and measurement errors, and a thorough statistical analysis should be conducted to determine the accuracy of the proposed algorithm.

\section{REFERENCES}

[1] L. Ljung, System identification - Theory for the User (2nd edition). Prentice Hall PTR, 1999.

[2] P. Van Overschee and B. De Moor, Subspace Identification for Linear Systems: Theory - Implementation - Applications. Kluwer Academic Publishers, 1996.

[3] T. Katayama, Subspace methods for system identification. Springer, 2006.

[4] M. Verhaegen and V. Verdult, Filtering and System Identification: A Least Squares Approach. Cambridge University Press, 2007.

[5] H. Kantz and T. Schreiber, Nonlinear Time Series Analysis (2nd edition). Cambridge University Press, 2004.

[6] F. Takens, "Detecting strange attractors in turbulence," in Dynamical Systems and Turbulence. Springer-Verlag, 1981, pp. 366381.

[7] H. D. I. Abarbanel and M. B. Kennel, "Local false nearest neighbors and dynamical dimensions from observed chaotic data," Phys. Rev. E, vol. 47, pp. 3057-3068, May 1993.

[8] C. Letellier, L. L. Sceller, G. Gouesbet, F. Lusseyran, A. Kemoun, and B. Izrar, "Recovering deterministic behavior from experimental time series in mixing reactor," AIChE Journal, vol. 43, no. 9, pp. 2194-2202, 1997.

[9] A. M. Dabroom and H. K. Khalil, "Discrete-time implementation of high-gain observers for numerical differentiation," Int. J. Control, vol. 72, no. 17, pp. 1523-1537, 1999.

[10] S. Diop, J. W. Grizzle, and F. Chaplais, "On numerical differentiation algorithms for nonlinear estimation," in Proc. 39th Conf. Decision Control, Sydney, Australia.

[11] S. Diop, J. W. Grizzle, P. E. Moraal, and A. Stefanopoulou, "Interpolation and numerical differentiation for observer design," in Proc. Amer. Control Conf., Baltimore, MD.

[12] A. Isidori, Nonlinear Control Systems (3rd edition). Springer, 1995.

[13] E. D. Sontag, Mathematical Control Theory: Deterministic Finite Dimensional Systems. Springer, 1998.

[14] A. Padoan and A. Astolfi, "Towards deterministic subspace identification for autonomous nonlinear systems," 54th Conf. Decision Control, Osaka, Japan, 2015, submitted. 

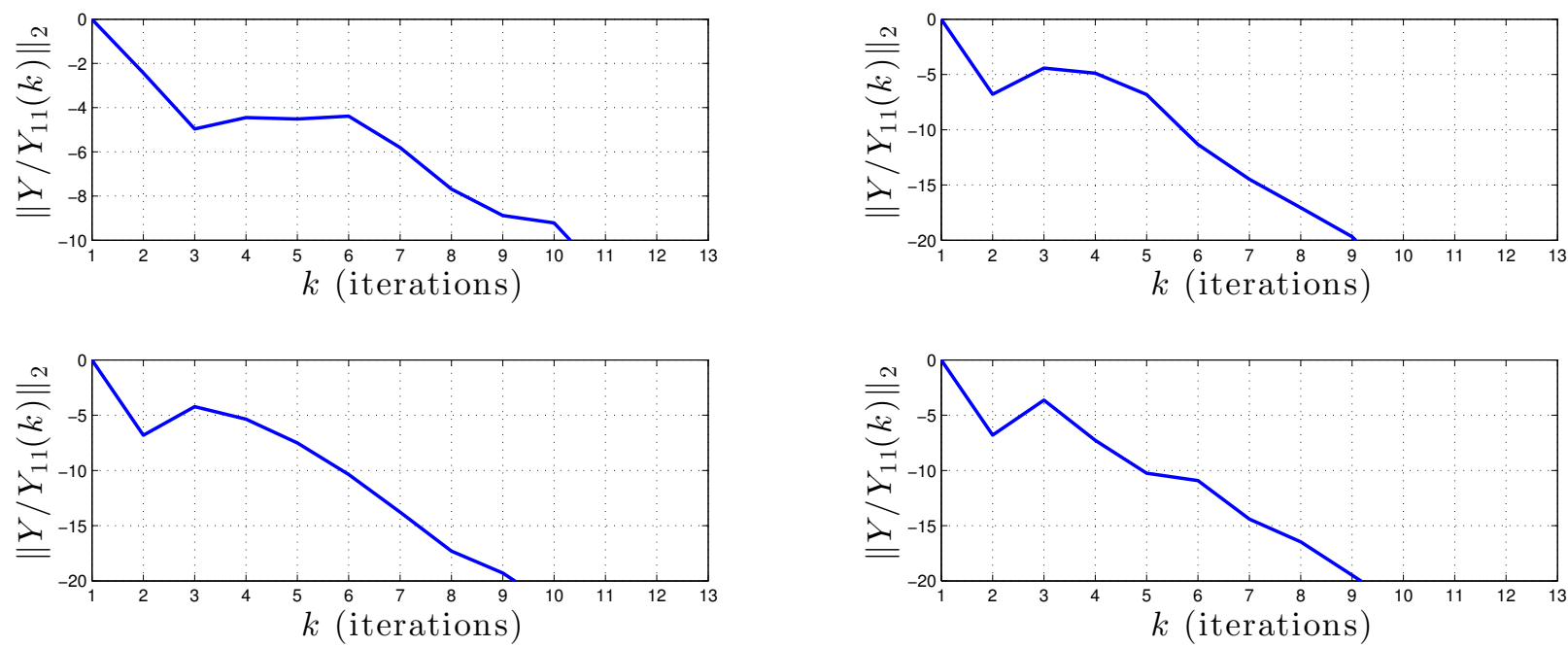

Fig. 1: The 2-norm $\left\|Y / Y_{11}(k)\right\|_{2}$ of the matrix $Y / Y_{11}(k)$ as a function of the iteration $k$ of Algorithm 1 for the system (5) (top-left), the Duffing oscillator (top-right), the pendulum (bottom-left), and the van der Pol oscillator (bottom-right).
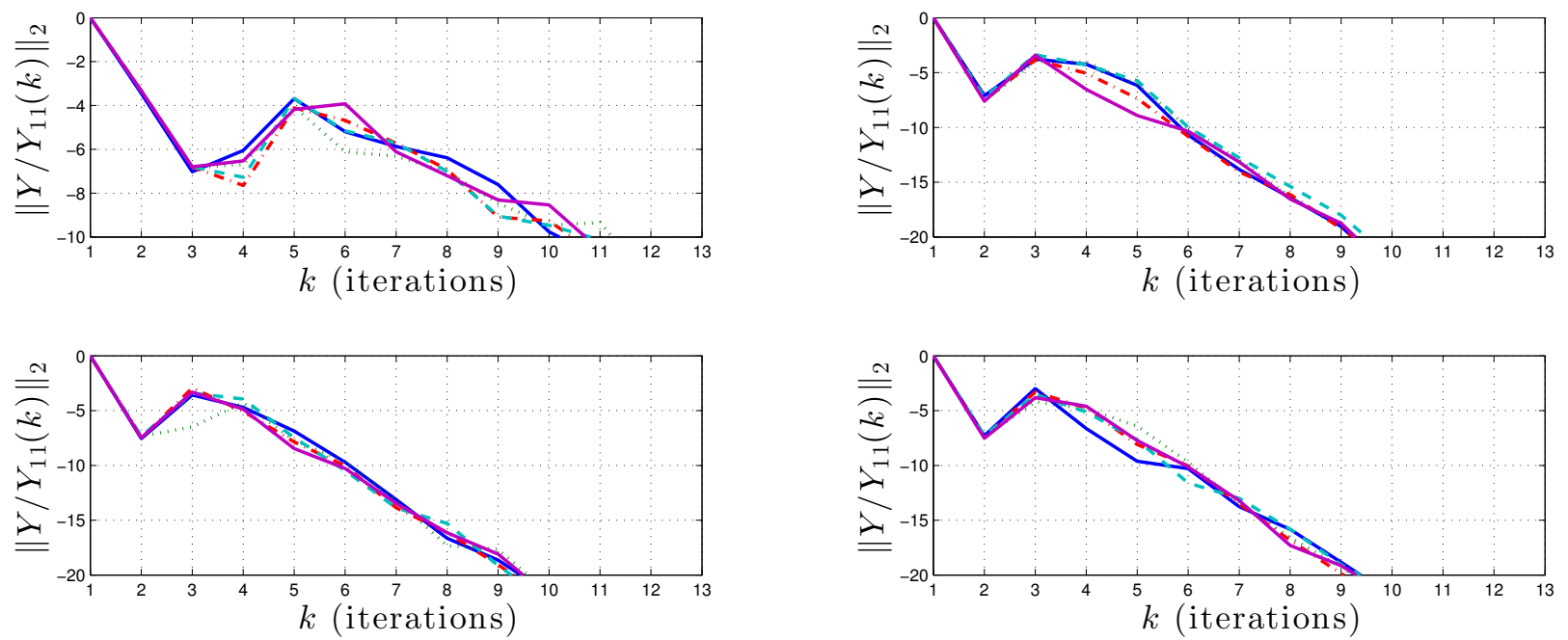

Fig. 2: The 2-norm $\left\|Y / Y_{11}(k)\right\|_{2}$ of the matrix $Y / Y_{11}(k)$ as a function of the iteration $k$ of Algorithm 1 on 5 consecutive time windows for the system (5) (top-left), the Duffing oscillator (top-right), the pendulum (bottomleft), and the van der Pol oscillator (bottom-right). 